Vallejo, M, Rojas, C. y Fernandez, A. (2002): Sesgos Relativos al Género en las Políticas Editoriales de Revistas Científicas Españolas del Campo de la Educación. RELIEVE:, v. 8, n. 2, p. 166-174.

http://www.uv.es/RELIEVE/v8n2/RELIEVEv8n2_3.htm

\title{
SESGOS RELATIVOS AL GÉNERO EN LAS POLÍTICAS EDITORIALES DE REVISTAS CIENTÍFICAS ESPAÑOLAS DEL CAMPO DE LA EDUCACIÓN
}

\section{(Gender Biases in Editorial Policy of Spaniard Scientific Journals in the Field of the Education)}

\begin{tabular}{ccc} 
& $p o r$ & \\
$\underline{\text { Article record }}$ & Mónica Vallejo Ruiz & $\underline{\text { Ficha del artículo }}$ \\
$\underline{\text { About authors }}$ & $\begin{array}{c}\text { Cristian Rojas Barahona y } \\
\text { HTML format }\end{array}$ & $\underline{\text { Sobre los autores }}$ \\
\hline
\end{tabular}

\begin{abstract}
This study analyses the status of gender which exists in the editorial teams or boards of Spanish journals in the field of educational. It also inquires into the possible bias relative to gender in the pyramid of eminence of Spanish educational researchers. As complementary analysis, the nationality or origin of the members of the editorial boards of the journals of education is considered. The main findings expose a strong bias against women when considering journal editors.
\end{abstract}

\section{Keywords}

Gender, editorial board, biases, scientific journals, Education, status of eminence Spain.

\section{Resumen}

Este estudio analiza el estatus del género existente en los equipos o consejos editoriales de las revistas científicas españolas de Educación. A priori, se indaga el estatus de la eminencia científica en el campo de la Educación; con el fin de detectar los posibles sesgos de género. Como análisis complementario se considera la nacionalidad o procedencia de los miembros de los consejos editoriales de las revistas de Educación. El hallazgo principal testimonia un fuerte sesgo contra las mujeres cuando éstas son consideradas editoras.

\section{Descriptores}

Género, consejos editoriales, sesgos, revistas científicas, educación, estatus de eminencia, España.

\section{Introducción}

Al hablar de sesgos podemos hacer referencia a tres niveles distintos en donde se ha hecho mención de ellos: primero está la Declaración Internacional de Filadelfia de 1994, en donde por primera vez se habla del principio de igualdad de oportunidades y de trato para hombres y mujeres; en segundo lugar el Convenio Europeo para la protección de los derechos humanos y de las libertades fundamentales de 1950, que en su artículo 14 recoge una genérica prohibición de la discriminación; y en último lugar, a nivel nacional, la Constitución Española, en su artículo-ley 1.1 de 1988, que eleva la igualdad a valor superior del Estado social y democrático de Derecho (Ballester, 1994). 
Vallejo, M, Rojas, C. y Fernandez, A. (2002): Sesgos Relativos al Género en las Políticas Editoriales de Revistas Científicas Españolas del Campo de la Educación. RELIEVE:, v. 8, n. 2, p. 166-174.

http://www.uv.es/RELIEVE/v8n2/RELIEVEv8n2 3.htm

Tras este proceso histórico, el estudio sobre el sesgo de género asume una preocupación capital en nuestra sociedad actual viéndose reflejado en un creciente interés por las investigaciones sobre dicho tema (Townsend, 1991; Uchida, 1991). Específicamente en este estudio nos centraremos en verificar si existe algún sesgo con respecto a altos cargos. Con relación a este tema, han aparecido a lo largo del tiempo numerosos trabajos que han avalado la presencia de tal sesgo de género; encontrándonos con trabajos como: Castaño, Iglesias, Mañas y Sánchez-Herrero (1999), que recogen según la categoría vigente de la Clasificación Nacional de Ocupaciones española (CON-94), que las mujeres ocupan el 31.6\% de los cargos directivos. Foullerat (2001), afirma que las mujeres ocupan las categorías más bajas dentro de su profesión, tanto en la empresa privada con un $0.6 \%$ de los puestos directivos, como en la administración pública con un $16 \%$ de los altos cargos. Rusch y Marshall (1995), demuestran la existencia de unos filtros que mantienen a las mujeres en zonas "marginales", como ellas mismas citan, de los puestos laborales.

Centrándonos en el objeto de nuestro estudio, en el campo de la investigación educativa, y en concreto en las revistas científicas españolas de Educación, desde la perspectiva del sesgo relativo a la valoración para el acceso a los puestos laborales en los consejos editoriales, analizaremos la importancia de la pertenencia de las mujeres en dichos consejos. Destacando la trascendencia del consejo editor por ser el organismo encargado de aceptar o rechazar cualquier publicación presentada, además de ser el organismo que define las políticas editoriales de la revista, constituyendo en el caso de revistas prestigiosas un lobby epistémico que contribuye al desarrollo de una disciplina científica (Fernández Cano, 1995, p. 65).

Con respecto a la influencia que pudiese tener el género de los componentes del consejo editorial se han realizado diversos estudios. Entre ellos se destacan: los estudios de Goldberg (1968); Paludi y Bauer (1983); y Paludi y Strayer (1984), que demuestran una clara tendencia de las mujeres a subvalorar el trabajo de las profe- sionales de su mismo sexo. Por su parte Moore (1978), aporta tres hallazgos afines:

1.- Que existe un sesgo negativo tanto en los revisores femeninos como masculinos sobre artículos escritos por hombres.

2.- Que los revisores de cada género encontraron más elementos positivos en libros escritos por autores del mismo género.

3.- Que existe una clara tendencia (no significativa) hacia un sesgo preferencial sobre el mismo género.

Por otro lado Lloyd (1990), observa en su investigación que las revisoras mujeres aceptan más artículos de autoras que de autores.

Por todo lo anteriormente expuesto muchas revistas educativas y psicológicas han ido generando cambios en sus políticas editoriales, tales como la revisión ciega, el aumento del número de revisoras y la promoción de la participación de las mujeres (APA, 2001).

Para que quede más claro lo que sería una política editorial no sexista, Fernández Cano (1995, p.88) describe los siguientes puntos:

1.- Requiere a los autores especificar el sexo de los participantes en un estudio.

2.- Realiza revisión ciega automática y no opcional.

3.- Emite instrucciones a los autores para que sigan las orientaciones para un lenguaje no exista, contenidas en el manual de publicación del APA (APA, 2001).

\section{Método}

\section{II.1. Problema.}

Tras el análisis cuantitativo de la presencia del género masculino y femenino en los equipos o consejos editoriales de las principales 
Vallejo, M, Rojas, C. y Fernandez, A. (2002): Sesgos Relativos al Género en las Políticas Editoriales de Revistas Científicas Españolas del Campo de la Educación. RELIEVE:, v. 8, n. 2, p. 166-174.

http://www.uv.es/RELIEVE/v8n2/RELIEVEv8n2 3.htm

revistas científicas españolas del ámbito de la Educación, revisaremos el posible sesgo sexista o de discriminación que pudiese existir para ocupar algún cargo dentro de dichos consejos editoriales.

Concretamente, este estudio se estructura en un doble análisis cuantitativo: por un lado, se han cuantificado los miembros del consejo de editores de las revistas españolas de Educación según género; y por otro, el profesorado universitario en servicio activo de todos los departamentos, a nivel nacional, relacionados con el ámbito de la Educación (según esta misma categoría). Esta última información ha sido obtenida a través del listado de profesores universitarios emitido por el Consejo de Universidades (MECD-Consejo de Universidades, 2001).

\section{II.2. Revisión de la literatura.}

Con relación al género de los componentes de los consejos directivos, existen acciones discriminatorias de sus miembros desde hace muchos años y desde diversas culturas (Rage, 2001). Jansen (1989), a este respecto, establece que la reproducción de modelos de poder y privilegio en la distribución social del conocimiento es debido a la ausencia del conocimiento crítico sobre género.

En un estudio escrito por Teghtsoonian (1974), concluye que existe a principios de los años 70 un evidente y creciente sesgo contra las mujeres hacia la pirámide de la eminencia. Su hallazgo final fue que las mujeres con una misma tasa de publicaciones que los hombres tienen menos posibilidades de ser reconocidas como editoras de una revista de investigación.

Posterior a este estudio, existen numerosas investigaciones, como la de Boice, Barlow, Johnson y Klosko (1984); Boice, Shaughness y Pesker (1985); De Meuse (1987); Fernández Cano (1995); Over (1981); Tryon (1981) y White (1985) que confirman las ideas de Teghtsoonian, pero a la vez expresan una tendencia positiva a la inclusión de la mujer en estos ámbitos.

\section{II.3. Diseño}

A la hora de especificar el método de investigación seguido se puede hacer sobre la base de distintos criterios: Con respecto a la metodología, este estudio se considera cuantitativo; con respecto al proceso formal seguido, es de tipo inductivo, pues a través de los datos recogidos se pretende hacer inferencias generales; y con respecto al objetivo de la investigación, es de tipo exploratorio descriptivo.

\section{II.4. Muestra}

La muestra utilizada en este estudio está constituida por 20 revistas científicas españolas del campo educativo; concretamente fueron utilizados los últimos números editados por tales revistas relativos al año 2001, de los que se analizaron las páginas referentes al consejo o equipo editorial. La selección de esas 20 revistas educativas se hizo en base al escalafonamiento valorativo contenido en el estudio de Fernández Cano y Bueno (2002).

El procedimiento seguido en este análisis cuantitativo, para determinar el género de los miembros de los consejos de las revistas fue a través de un patrón cultural, como es el nombre propio de cada uno de ellos (dicho resultados se encuentran en la tabla 1). Cabe mencionar, que para el cómputo total de miembros de los consejos se consideró cada uno de los cargos directivos de las revistas, es decir, director/a, subdirector/a, secretario/a, comité científico, consejo asesor, entre otros.

Una observación a destacar en este proceso de recogida de datos, fue la diversidad de designaciones relativas a dichos cargos directivos; encontrándose 19 denominaciones distintas para determinar el consejo editorial de la revista.

Tabla 1. Género de los Miembros del Equipo Editorial de Revistas Científicas Españolas de Educación 
Vallejo, M, Rojas, C. y Fernandez, A. (2002): Sesgos Relativos al Género en las Políticas Editoriales de Revistas Científicas Españolas del Campo de la Educación. RELIEVE:, v. 8, n. 2, p. 166-174.

http://www.uv.es/RELIEVE/v8n2/RELIEVEv8n2 3.htm

\begin{tabular}{|c|c|c|c|}
\hline Revistas & Hombres & Mujeres & $\begin{array}{l}\text { Total de } \\
\text { miembros }\end{array}$ \\
\hline Investigación en la Escuela & 37 & 11 & 48 \\
\hline Teoría de la Educación & 35 & 8 & 43 \\
\hline Revista de Educación Especial & 12 & 17 & 29 \\
\hline Cuadernos de Pedagogía & 2 & 3 & 5 \\
\hline Boletín de la Institución Libre de Enseñanza & 19 & 2 & 21 \\
\hline Revista Española de Pedagogía & 21 & 1 & 22 \\
\hline Bordón & 21 & 7 & 28 \\
\hline Infancia y Aprendizaje & 34 & 39 & 73 \\
\hline Revista de Educación & 24 & 11 & 35 \\
\hline Revista Interuniversitaria de Formación del Profesorado & 16 & 4 & 20 \\
\hline Revista de Investigación Educativa & 11 & 16 & 27 \\
\hline Revista de Didáctica de las Matemáticas. UNO & 24 & 14 & 38 \\
\hline Revista Complutense de Educación & 6 & 3 & 9 \\
\hline Revista de Ciencias de la Educación & 8 & 3 & 11 \\
\hline Revista de Historia de la Educación & 30 & 7 & 37 \\
\hline Revista Iberoamericana de Educación & 12 & 6 & 18 \\
\hline Revista Española de Orientación y Psicopedagogía & 22 & 18 & 40 \\
\hline Revista de Educación Universidad de Granada & 15 & 6 & 21 \\
\hline Aula de Innovación Educativa & 43 & 33 & 76 \\
\hline Enseñanza de las Ciencias & 39 & 8 & 47 \\
\hline Total & $\begin{array}{c}431 \\
(66,5 \%)\end{array}$ & $\begin{array}{c}217 \\
(33,5 \%)\end{array}$ & $\begin{array}{c}648 \\
(100 \%)\end{array}$ \\
\hline
\end{tabular}

III. Resultados

Para establecer un posible sesgo, con respecto a la mujer, en los consejos editoriales de las revistas educativas españolas; en un principio 
Vallejo, M, Rojas, C. y Fernandez, A. (2002): Sesgos Relativos al Género en las Políticas Editoriales de Revistas Científicas Españolas del Campo de la Educación. RELIEVE:, v. 8, n. 2, p. 166-174.

http://www.uv.es/RELIEVE/v8n2/RELIEVEv8n2 3.htm

debemos conocer si la base de la pirámide de la eminencia científica en Educación, está constituida de forma igualitaria o por el contrario, existe desde esta posición un sesgo con respecto a algún género determinado. Para ello, realizaremos un análisis cuantitativo a través de la razón hombre-mujer en todos aquellos departamentos universitarios relacionados con la Educación en el ámbito nacional.

Los datos obtenidos en este primer análisis cuantitativo quedan reflejados en la tabla número 2 .

Tabla 2. Razón Hombre-Mujer en el Ámbito Universitario de la Educación

\begin{tabular}{|l|c|c|}
\hline \multicolumn{1}{|c|}{ Departamentos } & Hombres & Mujeres \\
\hline \hline Métodos de Investigación y Diagnóstico en Educación & $112(56 \%)$ & $88(44 \%)$ \\
\hline \hline Didáctica y Organización Escolar & $254(48.8 \%)$ & $266(51.2 \%)$ \\
\hline \hline Teoría e Historia de la Educación & $202(58.7 \%)$ & $142(41.3 \%)$ \\
\hline \hline Psicología Evolutiva y de la Educación & $231(49.7 \%)$ & $234(50.3 \%)$ \\
\hline \hline Didáctica de las Matemáticas & $129(56.8 \%)$ & $98(43.2 \%)$ \\
\hline \hline Didácticas de las Ciencias Experimentales & $114(51.5 \%)$ & $107(48.5 \%)$ \\
\hline \hline Didáctica de las Ciencias Sociales & $82(46.6 \%)$ & $94(53.4 \%)$ \\
\hline \hline Didáctica de la Lengua y la Literatura & $139(40 \%)$ & $208(60 \%)$ \\
\hline \hline Didáctica de la Expresión Musical & $39(27.1 \%)$ & $105(72.9 \%)$ \\
\hline \hline Didáctica de la Expresión Corporal & $116(56.8 \%)$ & $88(43.2 \%)$ \\
\hline \hline \multicolumn{1}{|c|}{ Total } & $\mathbf{1 4 1 8 ( 4 9 . 8 \% )}$ & $\mathbf{1 4 3 0}(\mathbf{5 0 . 2 \% )}$ \\
\hline
\end{tabular}

Como se observa por esta tabla, globalmente, los departamentos de Educación están constituidos de forma igualitaria por hombres y mujeres, aunque existen una leve mayoría de mujeres. Lo que constata que, aquello que hemos denominado base de la pirámide de la eminencia, se conforma por un número igualitario de personas con relación al género.

Este primer hallazgo nos conduce a la segunda cuestión de investigación de una manera determinista, es decir, el análisis de los consejos editoriales va a determinar que en la cima de la pi- rámide de la eminencia, o por lo menos en un estrato superior, podamos hablar de sesgo o discriminación con respecto a la mujer o no.

Junto a esta información también pretendemos obtener el grado de presencia que reciben estas revistas por parte de miembros de otras nacionalidades, todo ello relacionado con el género de dichos miembros.

El análisis de estas revistas con respecto a las categorías citadas anteriormente quedan resumidas en la siguiente tabla (Tabla 3). 
Vallejo, M, Rojas, C. y Fernandez, A. (2002): Sesgos Relativos al Género en las Políticas Editoriales de Revistas Científicas Españolas del Campo de la Educación. RELIEVE:, v. 8, n. 2, p. 166-174.

http://www.uv.es/RELIEVE/v8n2/RELIEVEv8n2 3.htm

Tabla 3. Miembros de los Consejos Editoriales según Género y Nacionalidad

\begin{tabular}{|c|c|c||c|}
\hline & Hombres & Mujeres & Frecuencias marginales \\
\hline \hline Nacionales & $367(56.6 \%)$ & $190(29.3 \%)$ & $557(86 \%)$ \\
\hline \hline Extranjeros & $64(9.8 \%)$ & $27(4.1 \%)$ & $91(14 \%)$ \\
\hline \hline Total & $431(66.5 \%)$ & $217(33.5 \%)$ & $648(100 \%)$ \\
\hline
\end{tabular}

Figura 1. Porcentaje de hombres y mujeres en los consejos editoriales.

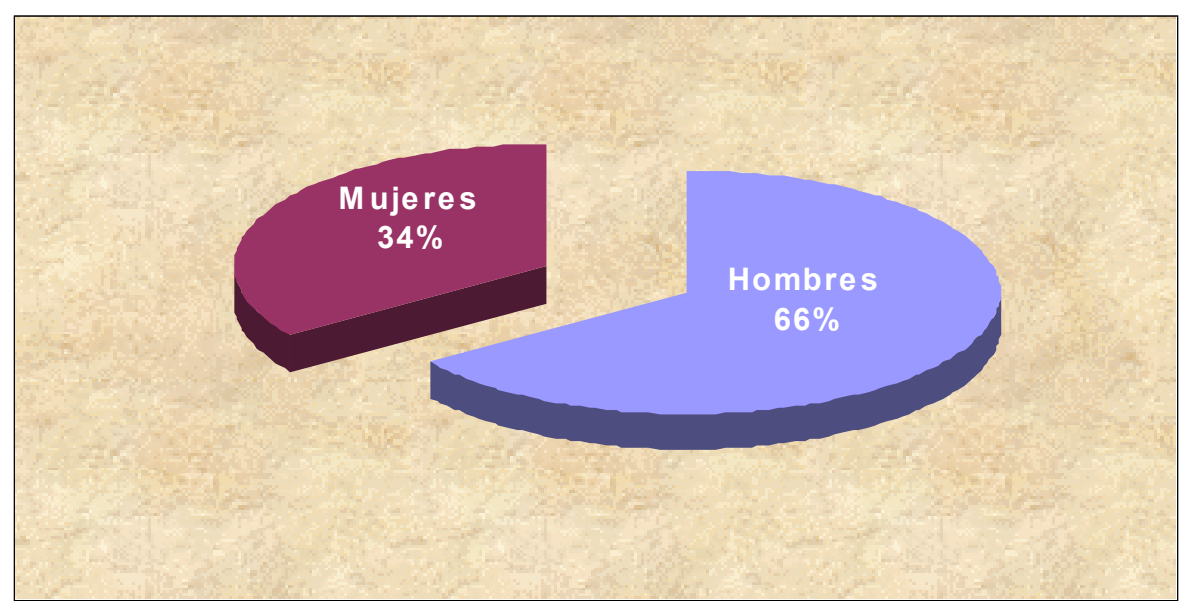

Como reflejan estos datos, tanto nacional como internacionalmente, existen un sesgo negativo con respecto a la inclusión de la mujer en los cargos editoriales o directivos de las revistas analizadas. Cuantificando este hallazgo, podríamos decir que los consejos editoriales de las revistas españolas de Educación están constituidas por el doble de hombres que de mujeres, es decir, una relación de dos por uno.

Otro de los hallazgos obtenidos es la escasa colaboración de miembros extranjeros en las revistas educativas, siendo aún menor la colaboración del género femenino. Este hallazgo nos lleva a definir nuestras revistas de Educación, según la conceptualización de White (1990), como "parroquianas"; es decir, la mayoría de sus componentes del consejo editorial son de la misma nacionalidad que la revista.

\section{Conclusiones}

Tras el análisis de los datos obtenidos, los hallazgos globales más destacados de este estudio son los siguientes:

1.- Existe una evidente igualdad de género en el campo laboral de la docencia universitaria española en el campo de la Educación (50\% $50 \%$ ), aunque se denota notables diferencias según departamentos.

2.- Se manifiesta la existencia de un sesgo relativo al género en los cargos directivos de los consejos editoriales de la revistas de Educación en España; sesgo negativo hacia el género femenino, tanto en el ámbito nacional como en el internacional. En concreto, existe una relación de dos hombres editores por una mujer editora.

3.- Con respecto a la nacionalidad de los integrantes que componen los consejos editoriales de las revistas analizadas, se evidencia 
Vallejo, M, Rojas, C. y Fernandez, A. (2002): Sesgos Relativos al Género en las Políticas Editoriales de Revistas Científicas Españolas del Campo de la Educación. RELIEVE:, v. 8, n. 2, p. 166-174.

http://www.uv.es/RELIEVE/v8n2/RELIEVEv8n2 3.htm

una supremacía de la nacionalidad española de sus miembros, existiendo una escasa participación de miembros extranjeros. Concretamente existe una relación de seis miembros nacionales por uno internacional.

\section{Recomendaciones}

Como aspecto complementario a las conclusiones presentadas, exponemos algunas sugerencias o recomendaciones que permitan profundizar o mejorar la problemática de sesgo de género en los consejos editoriales de las revistas españolas de Educación.

- Sería recomendable aumentar la presencia de mujeres nacionales y extranjeras en los consejos editoriales de las revistas, sin caer en el síndrome de la designación del cargo por imagen.

- Otra sugerencia sería ampliar el grado de colaboración de miembros extranjeros en los consejos editoriales de las revistas; promoviendo de este modo, revistas más internacionalistas con respecto a los miembros de su panel editorial.

- Sería conveniente hacer público los criterios de selección de los miembros de los consejos editoriales, mejorando la transparencia de las decisiones de selección de los mismos.

\section{Bibliografía}

APA, (2001). Publication Manual (5 $5^{\text {a }}$ Edición). Hyatsville, M.D.: APA.

Ballester, M. A. (1994). Diferencias y discriminación normativa por razón de sexo en el orden laboral. Valencia: Tirant Lo Blanch.

Boice, R.; Barlow, D.H.; Johnson, D. y Klosko, J. (1984). Behaviorists as peer reviewers: Do they misbehave?. Behavior Therapist, 7, 105107.

Boice, R.; Shaughness, P. y Pesker, T. (1985). Women and publishing in psychology. American Psychologist, 40, 577-578.

Castaño, C.; Iglesias, C.; Mañas, E.y SánchezHerrero, M. (1999). Diferencia o discriminación. La situación de las mujeres españolas en el mercado de trabajo y el impacto de las tecnolo- gías de la información. Madrid: Consejo Económico y Social.

De Meuse, K.P. (1987). A historical examination of author sex and research funding in industrial/organizational psychology. American Psychologist, 42, 876-879.

Fernández Cano, A. (1995). Métodos para evaluar la investigación en psicopedagogía. Madrid: Síntesis.

Fernández Cano, A. y Bueno A. (2002). Multivariate evaluation of Spanish educational research journal. Scientometrics, 55(1), 87-102.

Foullerat, F. (2001). Formación, orientación y género en educación. En T. Pozo, R. López Fuentes, B. García Lupión y E. Olmedo (Coords.), Investigación educativa: Diversidad y escuela (pp. 107-117). Granada: Grupo Editorial Universitario.

Goldberg, P. A. (1968). Are some women prejudiced against women? Transaction, 5 (5), 28-30.

Jansen, S. C. (1989). Gender and the information society: A socially structured silence. Journal of Communication, 39 (3),196-215.

Lloyd, M. E. (1990). Gender factors in reviewer recommendations for manuscript publication. Journal of Applied Behavior Analysis, 23 (4), 539-543.

MECD (2001). Consejo de Universidades. Listado de profesores Universitarios. Madrid: MECD.

Moore, M. (1978). Discrimination o favoritism? Sex bias in book reviews. American Psychologist, 33, 936-938.

Over, R (1982). Research productivity at impact of male at female psychologist. American Psychologist, 37, 24-31.

Paludi, M.A. y Bauer, W. D. (1983). Goldberg revisited: What's in an author's name?. Sex Roles, 9, 387-390.

Paludi, M.A. y Strayer, L. A. (1984). What's in an author's name? Differential evaluations of performance as a function of author's name. Sex Roles, 12, 353-361.

Rage, F. (2001). El silencio en la comunicación humana. Dossiers Feministes, 3, 88-98.

Rusch, E. y Marshall, C. (1995). The gender filters to the Work in the Administrative Culture. Annual Meeting of the American Educa- 
Vallejo, M, Rojas, C. y Fernandez, A. (2002): Sesgos Relativos al Género en las Políticas Editoriales de Revistas Científicas Españolas del Campo de la Educación. RELIEVE:, v. 8, n. 2, p. 166-174.

http://www.uv.es/RELIEVE/v8n2/RELIEVEv8n2 3.htm

tional Research of Association, San Francisco. ERIC- ED 392116

Teghtsoonian, M. (1974). Distribution by sex of authors at editors of psychological journals, 1970-72: Are there enough woman editors?. American Psychologist, 29, 262-269.

Townsend, B. K. (1991). The impact of feminist scholarship upon the study of higher education: An analysis of two higher education journals. ASHE Annual Meeting Paper. Annual Meeting of the Association for the Study of Higher Education, Boston. ERIC-ED 339330
Tryon, G. S. (1981). Publication records of women counsellors during the past decade. Journal of Counselling Psychology, 28, 184186.

Uchida, A. (1991). Gender politics: A review of journals and feminist scholarship. Annual Meeting of the Speech Communication Association, Atlanta. ERIC-ED 343174.

White, A. (1985). Women as authors and editors of psychological journal. American Psychologist, 40, 527-530.

White, R. T. (1990). Internationalism versus parochialism in educational research. The Review of Higher Education, 14 (1), 47-62.

\section{ABOUT THE AUTHORS / SOBRE LOS AUTORES}

Mónica Vallejo Ruiz (mailto:(mvruiz@,fedro.ugr.es): Licenciada en Pedagogía por la Universidad de Granada. Becaria FPU del Ministerio de Educación, Cultura y Deporte. Universidad de Granada. Sus principales líneas de trabajo son la evaluación de la investigación y cienciometría aplicada a Ciencias de la Educación.

Cristian Rojas Barahona (crojas@,fedro.ugr.es): Licenciado en Psicología. Trabaja en la Universidad Santo Tomás. Colegio de La Salle. Santiago de Chile. Sus principales líneas de trabajo son psicología cognitiva (razonamiento), psicología evolutiva y género

Antonio Fernández Cano (afcano@ugr.es): Profesor Titular del Departamento de Métodos de Investigación y Diagnóstico en Educación de la Universidad de Granada (Campus de la Cartuja -Granada). Teléfono:(+34) 9582442 53. Sus principales líneas de trabajo son la evaluación de la investigación y la cienciometría.

\section{ARTICLE RECORD / FICHA DEL ARTÍCULO}

\begin{tabular}{|l|l|}
\hline $\begin{array}{l}\text { Reference / } \\
\text { Referencia }\end{array}$ & $\begin{array}{l}\text { Vallejo, M.; Rojas, C. y Fernández Cano, A. (2002). Sesgos relativos al género en las políticas edito- } \\
\text { riales de revistas científicas españolas del campo de la educación. Revista ELectrónica de Investigación y } \\
\text { EValuación Educativa, v. 8, n. 2. http://www.Uv.es/RELIEVE/v8n2/RELIEVEv8n2_3.htm }\end{array}$ \\
\hline Title / Título & $\begin{array}{l}\text { Sesgos relativos al género en las políticas editoriales de revistas científicas españolas del } \\
\text { campo de la educación (Gender Biases in Editorial Policy of Spaniard Scientific Journals in } \\
\text { the Field of the Education). }\end{array}$ \\
\hline $\begin{array}{l}\text { Authors / Autores } \\
\text { Review / } \\
\text { Revista }\end{array}$ & Mónica Vallejo; Cristian Rojas y Antonio Fernández Cano \\
\hline ISSN & Revista ELectrónica de Investigación y EValuación Educativa (RELIEVE), v. 8, n. 2 \\
\hline $\begin{array}{l}\text { Publication date / } \\
\text { Fecha de publica- } \\
\text { ción }\end{array}$ & 2002 (Reception Date: 13 Feb. 2002; Publication Date: 10 October 2002) \\
\hline $\begin{array}{l}\text { Abstract / } \\
\text { Resumen }\end{array}$ & $\begin{array}{l}\text { Este estudio analiza el estatus del género existente en los equipos o consejos editoriales de } \\
\text { las revistas científicas españolas de Educación. A priori, se indaga el estatus de la eminencia }\end{array}$ \\
\hline
\end{tabular}


Vallejo, M, Rojas, C. y Fernandez, A. (2002): Sesgos Relativos al Género en las Políticas Editoriales de Revistas Científicas Españolas del Campo de la Educación. RELIEVE:, v. 8, n. 2, p. 166-174.

http://www.uv.es/RELIEVE/v8n2/RELIEVEv8n2 3.htm

\begin{tabular}{|l|l|}
\hline & $\begin{array}{l}\text { científica en el campo de la Educación; con el fin de detectar los posibles sesgos de género. } \\
\text { Como análisis complementario se considera la nacionalidad o procedencia de los miembros } \\
\text { de los consejos editoriales de las revistas de Educación. El hallazgo principal testimonia un } \\
\text { fuerte sesgo contra las mujeres cuando éstas son consideradas editoras }\end{array}$ \\
$\begin{array}{l}\text { This study analyses the status of gender which exists in the editorial teams or boards of } \\
\text { Spanish journals in the field of educational. It also inquires into the possible bias relative to } \\
\text { gender in the pyramid of eminence of Spanish educational researchers. As complementary } \\
\text { analysis, the nationality or origin of the members of the editorial boards of the journals of } \\
\text { education is considered. The main findings expose a strong bias against women when con- } \\
\text { sidering journal editors. }\end{array}$ \\
\hline $\begin{array}{l}\text { Keywords } \\
\text { Descriptores }\end{array}$ & $\begin{array}{l}\text { Género, consejos editoriales, sesgos, revistas científicas, Educación, estatus de eminencia, } \\
\text { España } \\
\text { Gender, editorial board, biases, scientific journals, Education, status of eminence, Spain }\end{array}$ \\
\hline $\begin{array}{l}\text { Institution / } \\
\text { Institución }\end{array}$ & Universidad de Granada (España) \\
\hline $\begin{array}{l}\text { Publication site / } \\
\text { Dirección }\end{array}$ & http://www.uv.es/RELIEVE \\
\hline Language / Idioma & Español (Title, abstract and keywords in english) \\
\hline
\end{tabular}

\section{Revista ELectrónica de Investigación y EValuación Educativa (RELIEVE)}

[ ISSN: 1134-4032 ]

(C) Copyright 2002, RELIEVE. Reproduction and distribution of this articles it is authorized if the content is no modified and their origin is indicated (RELIEVE Journal, volume, number and electronic address of the document).

// C Copyright 2002, RELIEVE. Se autoriza la reproducción y distribución de este artículo siempre que no se modifique el contenido y se indique su origen (RELIEVE, volumen, número y dirección electrónica del documento). 